\title{
Repeatability and sensitivity of FTIR ATR spectral analysis methods for bituminous binders
}

\author{
Bernhard Hofko • Mohammad Zia Alavi • Hinrich Grothe • David Jones • \\ John Harvey
}

Received: 27 March 2017/ Accepted: 7 June 2017/Published online: 10 June 2017

(C) The Author(s) 2017. This article is an open access publication

\begin{abstract}
Bituminous binders as organic materials are prone to aging mainly by oxidation. Aging changes the viscoelastic behavior of the material over time towards higher stiffness and brittleness. FTIR has been increasingly used lately to investigate impacts of oxidative aging on the chemical structure of bitumen. Especially the carbonyl and sulfoxide bands are affected by aging and commonly used to describe changes due to evolving oxidation of a binder. However, spectra obtained from FTIR can be analyzed in fundamentally different ways and the analysis method applied to a spectrum has an impact on the gathered results and especially on the repeatability and sensitivity with regards to oxidative changes. For the presented study, two bituminous binders, a PG 64-16 and an SBS modified PG 76-22 PM were aged by RTFO and PAV and subsequently measured by FTIR. The obtained spectra (up to 90 individual spectra per aging state) were analyzed employing various methods using either the original or a normalized spectrum, a band maximum or integration based calculation of indices from an absolute or tangential baseline. By analyzing the coefficient of variation of the different analysis methods, it was found that not all analysis
\end{abstract}

B. Hofko $(\bowtie) \cdot$ M. Z. Alavi · D. Jones · J. Harvey UCPRC, University of California, Davis, CA, USA e-mail: bernhard.hofko@tuwien.ac.at

H. Grothe

Institute of Materials Chemistry, Vienna University of Technology, Vienna, Austria methods exhibit the same repeatability and sensitivity. From the findings of the study it can be recommended to work with normalized spectra, use an absolute baseline and work with integration of areas for index production in favor over band maximum based methods.

Keywords Bitumen - Asphalt - Aging - Oxidation · Infrared spectroscopy · FTIR · Chemical composition

\section{Introduction}

Bituminous Binders extensively used in asphalt mixes for construction of pavements are mainly obtained from further processing of residuum of crude oil during refinery process. This material is well-considered as a very complex organic matter since it is composed of a large number of hydrocarbons and other elements (mainly $\mathrm{O}, \mathrm{N}, \mathrm{S}$, and traces of metals). The physical properties of bituminous binder, including its response to applied stress or strain, are highly temperature, time, and rate dependent, and thus, it is classified as a viscoelastic substance for mechanistic analysis [1-3]. From a chemical point of view, bituminous binders are highly sensitive to environmental non-load related actions, mostly oxidative aging $[4,5]$. The oxidative aging of asphalt binder occurs in two periods: in short-term during mix production while mixing binder and aggregates at 
elevated temperature (between 140 and $200{ }^{\circ} \mathrm{C}$, depending on binder type); and in long-term when an asphalt mix is in service over time in a pavement structure. Although mechanism of aging of bituminous binder is very complex, its consequences on physical and mechanical properties of binder are wellunderstood. Under sufficient supply of reactive oxygen species (ROS), the oxidative aging reaction causes the bituminous binder to become stiffer and more brittle, mostly agreed to be upon increasing association among polar components of binder. While the rheological behavior of bitumen and its change with aging, on the macro-level, has been extensively studied in the last decades [6-12] and sophisticated models have been developed to describe the complex behavior of this material [13-19], the chemical composition of bitumen, its changes with aging, and in-depth analysis of its impact on the mechanical behavior, is still subject to studying [20-24] and intrigue many researchers in chemistry, and civil materials engineering fields.

Several methods have been examined to analyze the chemistry of bituminous binders including the Corbett chromatography, Gas or Gel Permission Chromatography, Atomic Force Microscopy (AFM), Fourier Transform Infrared Spectroscopy (FTIR), etc. Amongst these techniques, FTIR has been increasingly used lately to investigate changes in the chemical composition due to oxidative aging. Recent FTIR devices do not require large and environmentally controlled lab space because of their compact design, require very small amount of sample for testing, and are time and cost efficient [25-34]. In general, the objective of infrared spectroscopy, as in FTIR, is to gain information on how much a given sample absorbs light (i.e. infrared radiation) at a certain wavelength range. Mid infrared radiation $\left(400-4000 \mathrm{~cm}^{-1}\right)$ excites vibrations of molecules which depend on their environment. Every linear and non-linear molecule has $3 \mathrm{~N}-5$ and $3 \mathrm{~N}-6$ normal vibrations, respectively; $\mathrm{N}$ is the number of atoms in this molecule. Only those vibrations are prone for infrared absorption where the dipole moment is changing due to the atomic movement. For this case, the energy of a photon is in resonance with the energy difference between a vibration ground level and the first excited vibrational state. The photon is canceled and the fundamental vibration is excited. This way, transmission absorption spectra can be recorded. However, for materials with extremely high absorption coefficients like bitumen, there is the inherent problem that the transmission is very low or impossible and thus the signal to noise ratio is unacceptable low. Reflectance spectroscopy provides remedy, since the radiation is reflected at the outer surface of the sample. Reflectance spectroscopy is accessible in three modes: diffuse reflectance infrared Fourier spectroscopy (DRIFTS), ranging angle infrared reflectance spectroscopy (RAIRS) and attenuated total reflectance (ATR). DRIFTS demands a powdered sample and relies on the particle scattering and refraction. RAIRS needs an absolutely plane film and highly specialized sample preparation. Thus, only ATR is the method of choice for bitumen. For ATR, an evanescent light wave is attenuated due to the resonances of molecular vibrations situated in the interface between the sample and a crystal of high refraction index (ZnSe, Cs, Si or diamond).

For spectra analysis derived from FTIR, different groups of molecular bounds can be unambiguously distinguished at different, well-defined wavelengths of an absorbance spectrum by characteristic band maxima (local maxima in the spectrum) [35].

For bituminous binders, especially those structures associated with oxidative aging, i.e. uptake of oxygen, have been in the focus of attention in research. Changes in these chemical groups can then be correlated to changes in the rheological properties and allow for a better understanding of chemomechanical coupling [36].

For analysis of the obtained spectra, different approaches can be used. Spectra can be used in their original form and they can be normalized prior to analysis, respectively. It is possible to either look at single values from absorbance band maxima or to consider a range of wavenumbers by integrating the area below an absorbance spectrum around a certain band. In addition, the basis for obtaining the value can either be an absolute one (absorbance value of zero) or a relative one, i.e. a tangential base line.

\subsection{Objective and methodology}

The objective of this study is to give a recommendation on, which of the different methods for spectra analysis to use in order to obtain highly repeatable results for changes in the oxidative structures of bituminous binders upon aging. In addition, the recommendation take into account which of the 
analysis methods gives most sensitive results, i.e. which method is able to detect small changes in the chemical structures due to oxidation with high precision. To reach this goal, the following methodology is applied:

- Prepare different oxidative aging states of bituminous binder samples by applying standardized aging methods (RTFO and PAV);

- Run FTIR-ATR (attenuated total reflection) on individual samples in the different aging states;

- Analyze the obtained spectra with different analysis methods;

- Calculate defined indices for the oxidized structures (carbonyl and sulfoxide) and derive basic statistical information for these indices;

- Compare repeatability and sensitivity of these indices for each applied analysis method to produce recommendations based on a sound sample size.

\section{Materials and methods}

\subsection{Materials}

The study was carried out with two types of binders commonly used in road pavements: an unmodified PG 64-16 binder and an SBS modified PG 76-22 PM. The last mentioned was used for validation purposes. The binders were supplied from two different refineries located in northern California. The samples were analyzed in its unaged and RTFO aged state, as well as after PAV aging for 20, 40 and $60 \mathrm{~h}$.

\subsection{Binder aging}

Each binder was aged by RTFO according to EN 12607-1 [37] at $163{ }^{\circ} \mathrm{C}$ for $75 \mathrm{~min}$ to bring the material into a state of short-term aging. Parts of the RTFO aged sample were further aged by PAV according to EN 14769 [38] at $100{ }^{\circ} \mathrm{C}$ and $2.1 \mathrm{MPa}$ for 20,40 and $60 \mathrm{~h}$, to simulate aging in longer terms as years in pavement service life.

\subsection{FTIR ATR}

For FTIR ATR analysis, all binder samples were preheated in sealed containers, each carrying about
$20 \mathrm{~g}$ of binder, at $163{ }^{\circ} \mathrm{C}$ for no longer than $10 \mathrm{~min}$ to be sufficiently workable for sample preparation. The preheating was kept as short as possible to prevent any further change in chemical composition of binder. After thoroughly cleaning the optics with an organic solvent (Orange Solve Plus [39]) to dissolve and remove any remaining binder and acetone to remove any remaining solvent, a small mass of preheated binder was taken by a spatula and then applied directly onto the FTIR optics. To make sure that the sample was in direct contact with the optics throughout the analysis, a paper spacer was applied on top of the sample and a constant pressure was applied to the paper spacer by the holding arm of the device.

The spectra were measured by a Bruker ALPHA FTIR Spectrometer with a Platinum ATR that holds a diamond ATR module. Thus, the spectra were recorded in reflective mode from 4000 to $400 \mathrm{~cm}^{-1}$ at a resolution of $4 \mathrm{~cm}^{-1}$ averaging 24 scans for each measurement. The sample was carefully removed from the optics using a spatula, followed by cleaning the optics again with the solvent and acetone before applying the next sample.

10 individual samples of the PG 64-16 were analyzed by FTIR ATR for each state of aging. For each of these samples, 9 individual spectra were recorded. Thus a total of 450 spectra were employed for this study, 90 spectra for each state of aging. For the PG 76-22 PM, 5 individual samples were analyzed for each state of aging to validate the findings of the PG 64-16. Again, 9 individual spectra were recorded for each sample, resulting in 45 spectra for each state of aging.

All spectra were analyzed to obtain information on changes in the chemical composition by oxidative aging. Therefore, changes in the carbonyl and sulfoxide groups were taken into consideration.

\subsubsection{Spectral analysis approaches}

To assess different ways of FTIR ATR spectral analysis, three basic approaches were systematically combined and compared.

(a) Spectra were looked at with or without further normalization. In case no normalization was applied (=original spectra), the data from oxidized groups were analyzed relative to a reference group. The aliphatic group (symmetric and 
asymmetric bending vibrations around 1460 and $1376 \mathrm{~cm}^{-1}$, respectively) is commonly used as a reference group, since it is anticipated that these structures are stable and not affected by applied aging procedures [40].

In case of normalization of the spectra prior to analysis, the asymmetric stretching vibration at of the aliphatic structures $2923 \mathrm{~cm}^{-1}$ was taken as the basis for normalization. For normalization, the absorbance value of this band was set to 1.0 and the complete spectrum was multiplied by a ratio factor. The normalization is based on the equation below:

$a_{\mathrm{norm}}(w)=a(w) \cdot \frac{1}{a\left(2923 \mathrm{~cm}^{-1}\right)}$

with $a_{\text {norm }}(w)$, normalized absorbance spectrum as a function of wave number $w ; a(w)$, original absorbance spectrum; $a\left(2923 \mathrm{~cm}^{-1}\right)$, original absorbance value at the reference band at wave number $2923 \mathrm{~cm}^{-1}$.

Both approaches, one based on original spectra with reference groups and the other one with normalized spectra are based on the idea to remove any variation in the absorbance spectra due to a variation of the IR beam penetration between samples, which would bias further interpretation of results. (b) The considered structural groups were then either looked at by band maxima or integration analysis. For the band analysis, just a single absorbance value of the band maximum of a certain group is taken into account. For the integration analysis, the area below the absorbance spectrum around a peak is considered.

(c) For both cases, band and integration analysis, the basis for band height or integration area can either be the absolute base line at an absorbance value of 0 , or a tangential approach can be taken. For the tangential approach, a relative base line is chosen for each band in a way that the relative base line approaches the spectrum in a tangential way.

An FTIR ATR example spectrum (original and normalized) indicating all considered structural groups and showing examples of base and tangential band and integration analysis is presented in Fig. 1. It shows the spectrum of an RTFO + PAV aged sample.

From the three basic approaches described above, eight different ways of spectral analysis can be obtained and were considered for this research. They are summarized in Table 1.

The following parameters are defined for the different analysis approaches:
Fig. 1 Example of FTIR ATR absorbance spectrum for an unmodified RTFO + PAV aged binder sample




Table 1 Considered spectral analysis methods

\begin{tabular}{|c|c|c|c|c|c|c|}
\hline & \multicolumn{2}{|l|}{ Index } & \multicolumn{2}{|l|}{ Baseline } & \multicolumn{2}{|l|}{ Spectrum } \\
\hline & Band position & Integration & Absolute baseline & Tangential baseline & Original & Normalized \\
\hline IBR & & $\mathrm{x}$ & $\mathrm{x}$ & & $\mathrm{x}$ & \\
\hline ITR & & $\mathrm{x}$ & & $\mathrm{x}$ & $\mathrm{x}$ & \\
\hline PBR & $\mathrm{x}$ & & $\mathrm{x}$ & & $\mathrm{x}$ & \\
\hline PTR & $\mathrm{x}$ & & & $\mathrm{x}$ & $\mathrm{x}$ & \\
\hline IBN & & $\mathrm{x}$ & $\mathrm{x}$ & & & $\mathrm{x}$ \\
\hline ITN & & $\mathrm{x}$ & & $\mathrm{x}$ & & $\mathrm{x}$ \\
\hline $\mathrm{PBN}$ & $\mathrm{x}$ & & $\mathrm{x}$ & & & $\mathrm{x}$ \\
\hline PTN & $\mathrm{x}$ & & & $\mathrm{x}$ & & $\mathrm{x}$ \\
\hline
\end{tabular}

$$
\begin{aligned}
& \mathrm{PB}_{i}=\left.\max a(w)\right|_{\mathrm{PB}_{\text {norm }, i}=\max a_{\text {norm }}(w)||^{w_{l, i}}} ^{w_{u, i}} w_{l, i} \\
& \mathrm{IB}_{i}=\int_{w_{l, i}}^{w_{u, i}} a(w) \mathrm{d} w \\
& \mathrm{IB}_{\text {norm }, i}=\int_{w_{l, i}}^{w_{u, i}} a_{\text {norm }}(w) \mathrm{d} w
\end{aligned}
$$

with $a(w)$, absorbance value at wavenumber $w$; $a_{\text {norm }}(w)$, normalized absorbance value at wavenumber $w ; w_{u, i}$, upper wavenumber limit for structural group $i ; w_{l, i}$, lower wavenumber limit for structural group $i ; \mathrm{PB}_{i}$, band maximum from absolute base line for structural group $i$; $\mathrm{PB}_{\text {norm }, i}$, normalized band maximum from absolute base line for structural group $i$; $\mathrm{IB}_{i}$, integrated area from absolute base line for structural group $i$; $\mathrm{IB}_{\text {norm }, i}$, normalized, integrated area from absolute base line for structural group $i$, and

$$
\begin{aligned}
& \mathrm{PT}_{i}=\max a(w) \mid \begin{array}{l}
w_{u, i} \\
w_{l, i}
\end{array} \\
& -\left(a\left(w_{u, i}\right)-\frac{a\left(w_{u, i}\right)-a\left(w_{l, i}\right)}{w_{u, i}-w_{l, i}} \cdot\left(w_{u, i}-w_{\max , \mathrm{i}}\right)\right) \\
& \mathrm{PT}_{\mathrm{norm}, i}=\max a_{\text {norm }}(w) \mid \begin{array}{l}
w_{u, i} \\
w_{l, i}
\end{array} \\
& -\left(a_{\mathrm{norm}}\left(w_{u, i}\right)-\frac{a_{\mathrm{norm}}\left(w_{u, i}\right)-a_{\mathrm{norm}}\left(w_{l, i}\right)}{w_{u, i}-w_{l, i}}\right. \\
& \left.\cdot\left(w_{u, i}-w_{\max , i}\right)\right)
\end{aligned}
$$

$$
\begin{aligned}
& \mathrm{IT}_{i}=\int_{w_{l, i}}^{w_{u, i}} a(w) \mathrm{d} w-\frac{a\left(w_{u, i}\right)+a\left(w_{l, i}\right)}{2} \cdot\left(w_{u, i}-w_{l, i}\right) \\
& \mathrm{IT}_{\mathrm{norm}, i}=\int_{w_{l, i}}^{w_{u, i}} a_{\mathrm{norm}}(w) \mathrm{d} w-\frac{a\left(w_{u, i}\right)+a\left(w_{l, i}\right)}{2} \cdot\left(w_{u, i}-w_{l, i}\right)
\end{aligned}
$$

with $w_{\max , i}$, wavenumber of band maximum for structural group $i ; \mathrm{PT}_{i}$, band maximum from tangential base line for structural group $i ; \mathrm{PT}_{\text {norm }, i}$, normalized band maximum from tangential base line for structural group $i$ : $\mathrm{IT}_{i}$, integrated area from tangential base line for structural group $i$; $\mathrm{IT}_{\text {norm }, i}$, normalized, integrated area from tangential base line for structural group $i$.

The structural groups $i$ can be the carbonyl group $(\mathrm{C}=\mathrm{O})$, the sulfoxide group $(\mathrm{S}=\mathrm{O})$ or the reference group (ref).

For each of the derived spectra the following 8 indices were calculated:

$$
\begin{aligned}
\mathrm{PBR}_{i}=\frac{\mathrm{PB}_{i}}{\mathrm{~PB}_{\text {ref }}} & \mathrm{IBR}_{i}=\frac{\mathrm{IB}_{i}}{\mathrm{IB}_{\text {ref }}} \\
\mathrm{PBN}_{i}=\mathrm{PB}_{\text {norm }, i} & \mathrm{IBN}_{i}=\mathrm{IB}_{\text {norm }, i} \\
\mathrm{PTR}_{i}=\frac{\mathrm{PT}_{i}}{\mathrm{PT}_{\text {ref }}} & \mathrm{ITR}_{i}=\frac{\mathrm{IT}_{i}}{\mathrm{IT}_{\text {ref }}} \\
\mathrm{PTN}_{i}=\mathrm{PT}_{\text {norm }, i} & \mathrm{ITN}_{i}=\mathrm{IT}_{\text {norm }, i}
\end{aligned}
$$

Since for each aging state, 10 individual samples were analyzed with 9 spectra per sample, a total of sample size of 90 data items were derived for each aging state. Mean value (MV), standard deviation (SD) and coefficient of variance (CV) were calculated for each index. 
Fig. 2 FTIR ATR spectra of PG 64-16 from all aging states



\section{Results and interpretation}

Section 3.1 investigates changes of the oxidized structures in the PG 64-16 binder in detail and assesses repeatability and sensitivity of different analysis methods based on PG 64-16 results. Section 3.2 presents a validation of this assessment based on the PG 76-22 PM binder.

\subsection{Analysis of PG 64-16 samples}

The upper and lower wave numbers for the structural groups of PG 64-16 were defined as follows:

- Carbonyl: $1666-1746 \mathrm{~cm}^{-1}$

- Sulfoxide: $924-1066 \mathrm{~cm}^{-1}$

- Reference (aliphatic): $1319-1520 \mathrm{~cm}^{-1}$

Figure 2 shows an example of normalized FTIR ATR spectra from PG 64-16 of the five considered states of aging from unaged to PAV aged at $100{ }^{\circ} \mathrm{C}$ for $60 \mathrm{~h}$ from wavenumbers $600-2000 \mathrm{~cm}^{-1}$. The vertical marker at $1680 \mathrm{~cm}^{-1}$ indicates the position of the carbonyl band and the marker at $1030 \mathrm{~cm}^{-1}$ the position of the sulfoxide band. With an increase in aging, the carbonyl area slightly increases from unaged to RTFO aged and then strongly with clearly visible band maximums for the PAV aged states. The situation of the sulfoxide area is different. Sulfoxides are already present in most unaged bituminous binders. A clear signal is visible in Fig. 2. The sulfoxides are usually decreased upon RTFO aging, before strongly increasing again for PAV aged samples.

To analyze the changes in carbonyl and sulfoxide index in more detail and depending on the applied analysis method, Table 2 shows the carbonyl index for all aging states and all eight analysis methods in terms of MV and SD.

In general, all analysis methods agree that the carbonyl index continuously increases with aging. For the three PAV aged samples with different aging duration, it seems that the increase follows a linear trend. Those methods that use the absolute base line ("B") for analysis purposes exhibit a carbonyl index even for the unaged binder state. This of course is an artificial effect since no peak is actually visible in the spectra. The effect can be prevented when a tangential base line ("T") is used. Indices from these methods also confirm the visual analysis that hardly any carbonyl structures are produced upon RTFOT aging, since the index is small to negligible for RTFOT samples.

Analysis methods that use the same baseline approach and same index (band maximum or integration) and differ only in terms of spectra (original vs. normalized) show congruent evolution of indices with aging (e.g. compare IBR with IBN). The difference between methods is the scale. The integration based 




method from absolute baseline using the normalized spectrum shows carbonyl indices that are about the factor 100 larger (IBR vs. IBN). The integration based method with tangential baseline from normalized spectrum shows about 40 times larger indices than the method from original spectrum (ITR vs. ITN). No relevant scale effect is evident for the band maximum based methods.

Most of the statements made for carbonyl index in Table 2 can be transferred to Table 3 , which shows the sulfoxide indices for all methods and states of aging. However, the evolution of the sulfoxide index with aging is different. All analysis methods agree that RTFOT aging leads to a reduction in sulfoxides, the reduction can be most prominently seen for the tangential based methods (ITR, ITN, PTR and PTN). All four absolute baseline based methods are consistent in showing that the sulfoxides increase rather linearly with increasing PAV aging time, whereas the tangential based methods show a minimum for $40 \mathrm{~h}$ PAV aging. This could be a biased effect from an inappropriate choice of wave length limits. However, a parametric study in which these limits were systematically varied showed no effect of the wave length limits on the principle trends.

\subsubsection{Repeatability}

To investigate and rank the repeatability of the eight considered analysis methods, the coefficient of variation $(\mathrm{CV})$ or relative standard deviation was used as a benchmark parameter. The smaller the $\mathrm{CV}$ is the smaller the scatter of results (in terms of standard deviation) relative to the mean value of a sample. Thus, a small CV correlates with a high repeatability if the sample size is large enough. In this case, the sample size $n$ is 90 for each binder sample, since 90 spectra were analyzed per binder from 10 individual samples per binder.

Table 4 contains the CVs for all aging states and analysis methods for the carbonyl index in the upper part of the table and the sulfoxide index in the lower part of the table, respectively. The CV is given for each aging state with the exception of the unaged state of carbonyl index for the four methods using the tangential approach. For these four methods the mean value of the carbonyl index is too close to zero to calculate sensible CVs. In addition, the tables contain the mean $\mathrm{CV}$ for a method considering all aging states. 




Bold numbers in the tables show the smallest $\mathrm{CV}$ for a case. The row highlighted grey shows the analysis method with smallest mean CV.

For both, the carbonyl and sulfoxide indices, the smallest $\mathrm{CV}$, i.e. best repeatability can be found for the integration and absolute baseline based method when the original spectrum is taken into consideration (IBR, $3.0 \%$ for $\mathrm{C}=\mathrm{O}$ and $1.6 \%$ for $\mathrm{S}=\mathrm{O}$ ) and for the band maximum and absolute baseline base method when the normalized spectrum is considered ( $\mathrm{PBN}, 4.8 \%$ $\mathrm{C}=\mathrm{O}$ and $2.7 \% \mathrm{~S}=\mathrm{O}$ ). However, the BPR method and the IBN method show only slightly higher mean CVs.

The methods based on integration from a tangential baseline show the worst repeatability for use of original and normalized spectrum (ITR and ITN).

The findings can be underlined by a theoretical consideration of error evolution with increasing number of data points. Considering that $e$ is the error attached to one measurement, i.e. one data point in the absorbance spectrum, the error attached to the average of $n$ data points is the error (standard deviation) divided by the square root of $n$. The higher the number of data points $n$ taken into account is, the lower is the resulting error. Applying this concept to the considered analysis methods, the following number of data points is taken into account by for each option of the methods:

- P: 1 data point, i.e. the band maximum

- I: $n$ data points, i.e. number of data points between lower and upper wavenumber limit for integration

- B: $n$ data points, i.e. the absolute base line between lower and upper wavenumber limit

- T: 2 data points, i.e. the two limits in the absorbance spectrum used for tangential methods.

Since the repeatability analysis was carried out separately for methods using original (R) and normalized $(\mathrm{N})$ spectra, these two options are not taken into account for the error consideration. Assuming further that $n$ is considerably larger than 2, methods IBx $(n+n$ data points) should exhibit the smallest error (smallest CV, best repeatability), followed by methods ITx $(n+2)$, PBx $(n+1)$ and PTx $(1+2)$. This is partly confirmed by the CV values in Table 4 . IBx methods are among the smallest CVs in every case. However, ITx methods show highest CVs in all cases. It seems that methods based on tangential base lines (T) are more sensitive to errors in the tangential end 
Table 4 Coefficient of variation of carbonyl and sulfoxide indices of PG 64-16 for all analysis methods

\begin{tabular}{|c|c|c|c|c|c|c|}
\hline $\mathrm{C}=\mathrm{O}$ & unaged & RTFOT & PAV 10020 & PAV 10040 & PAV 10060 & mean $\mathrm{CV}$ \\
\hline IBR & $6.4 \%$ & $2.1 \%$ & $3.1 \%$ & $3.3 \%$ & $3.7 \%$ & $3.0 \%$ \\
\hline ITR & $\mathrm{N} / \mathrm{A}$ & $37.0 \%$ & $10.9 \%$ & $8.3 \%$ & $6.0 \%$ & $15.6 \%$ \\
\hline PBR & $4.8 \%$ & $1.9 \%$ & $3.5 \%$ & $3.8 \%$ & $3.8 \%$ & $3.3 \%$ \\
\hline PTR & $\mathrm{N} / \mathrm{A}$ & $\mathrm{N} / \mathrm{A}$ & $9.7 \%$ & $7.1 \%$ & $5.3 \%$ & $7.4 \%$ \\
\hline IBN & $5.9 \%$ & $4.4 \%$ & $7.3 \%$ & $5.7 \%$ & $4.6 \%$ & $5.5 \%$ \\
\hline ITN & $\mathrm{N} / \mathrm{A}$ & $37.0 \%$ & $10.7 \%$ & $8.1 \%$ & $5.7 \%$ & $15.4 \%$ \\
\hline $\mathrm{PBN}$ & $4.3 \%$ & $3.1 \%$ & $6.6 \%$ & $5.5 \%$ & $4.0 \%$ & $4.8 \%$ \\
\hline $\mathrm{PTN}$ & $\mathrm{N} / \mathrm{A}$ & $\mathrm{N} / \mathrm{A}$ & $9.6 \%$ & $7.1 \%$ & $5.4 \%$ & $7.3 \%$ \\
\hline $\mathrm{S}=\mathrm{O}$ & unaged & RTFOT & PAV 10020 & PAV 10040 & PAV 10060 & mean $\mathrm{CV}$ \\
\hline IBR & $5.0 \%$ & $1.8 \%$ & $1.9 \%$ & $1.7 \%$ & $1.2 \%$ & $1.6 \%$ \\
\hline ITR & $20.6 \%$ & $13.4 \%$ & $5.3 \%$ & $3.1 \%$ & $1.5 \%$ & $5.8 \%$ \\
\hline PBR & $8.5 \%$ & $2.1 \%$ & $2.5 \%$ & $2.0 \%$ & $1.3 \%$ & $2.0 \%$ \\
\hline PTR & $21.1 \%$ & $6.7 \%$ & $4.8 \%$ & $3.1 \%$ & $1.5 \%$ & $4.0 \%$ \\
\hline IBN & $5.0 \%$ & $2.1 \%$ & $4.1 \%$ & $3.6 \%$ & $2.3 \%$ & $3.0 \%$ \\
\hline ITN & $20.6 \%$ & $13.4 \%$ & $5.1 \%$ & $2.9 \%$ & $1.5 \%$ & $5.7 \%$ \\
\hline $\mathrm{PBN}$ & $8.0 \%$ & $2.0 \%$ & $4.0 \%$ & $3.1 \%$ & $1.8 \%$ & $2.7 \%$ \\
\hline $\mathrm{PTN}$ & $21.0 \%$ & $6.7 \%$ & $4.6 \%$ & $2.9 \%$ & $1.5 \%$ & $3.9 \%$ \\
\hline
\end{tabular}

Bold numbers show the smallest $\mathrm{CV}$ for a case. The rows highlighted grey show the analysis method with smallest mean $\mathrm{CV}$

points when combined with integration (I) rather than with band maxima (B).

\subsubsection{Sensitivity}

To investigate the sensitivity of different analysis methods to changes in the carbonyl and sulfoxide index, a sensitivity index (SI) is introduced. For this SI, only data from PAV aged samples is taken into account. It is assumed that the change in the carbonyl and sulfoxide index can be approximated by a linear regression that links the index with the PAV aging duration. Figure 3 shows an example for the IBN, the carbonyl index on the left and the sulfoxide index on the right. The larger the slope of the linear regression and the smaller the mean $\mathrm{CV}$ for a given analysis method is, the more significant are changes in the index and thus, the more sensitive is the given analysis method. The sensitivity index is therefore defined as follows:

$$
\mathrm{SI}_{i, k}=\frac{b_{i, k}}{\overline{\mathrm{CV}_{i, k}}}
$$

Fig. 3 Evolution of carbonyl index (left) and sulfoxide index (right) of PG 64-16 with PAV duration for IBN method
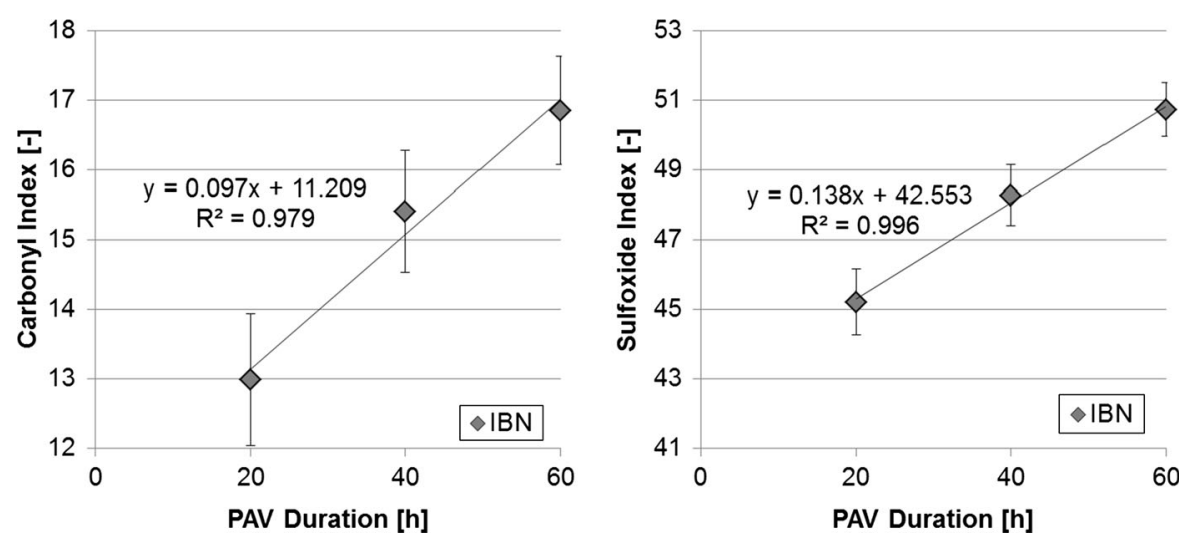
Table 5 Sensitivity index of carbonyl and sulfoxide indices of PG 64-16 for all analysis methods

\begin{tabular}{|c|c|c|c|c|c|c|c|c|}
\hline & \multicolumn{4}{|c|}{ Carbonyl Index } & \multicolumn{4}{|c|}{ Sulfoxide Index } \\
\hline & Slope $b_{C O}$ & $\mathrm{R}^{2}$ & mean CV & $\begin{array}{c}\text { Sensitivity } \\
\text { Index }\end{array}$ & Slope $b_{\text {so }}$ & $\mathrm{R}^{2}$ & mean $\mathrm{CV}$ & $\begin{array}{l}\text { Sensitivity } \\
\text { Index }\end{array}$ \\
\hline IBR & 0.00088 & 0.991 & $3.0 \%$ & 0.029 & 0.00100 & 0.997 & $1.6 \%$ & 0.061 \\
\hline ITR & 0.00099 & 0.989 & $15.6 \%$ & 0.006 & 0.00061 & 0.688 & $5.8 \%$ & 0.010 \\
\hline PBR & 0.00158 & 0.986 & $3.3 \%$ & 0.048 & 0.00098 & 0.942 & $2.0 \%$ & 0.049 \\
\hline PTR & 0.00124 & 0.980 & $7.4 \%$ & 0.017 & 0.00027 & 0.215 & $4.0 \%$ & 0.007 \\
\hline IBN & 0.09681 & 0.979 & $5.5 \%$ & 1.761 & 0.13797 & 0.996 & $3.0 \%$ & 4.565 \\
\hline ITN & 0.03932 & 0.989 & $15.4 \%$ & 0.256 & 0.02180 & 0.614 & $5.7 \%$ & 0.382 \\
\hline PBN & 0.00173 & 0.978 & $4.8 \%$ & 0.036 & 0.00115 & 0.972 & $2.7 \%$ & 0.042 \\
\hline PTN & 0.00104 & 0.980 & $7.3 \%$ & 0.014 & 0.00021 & 0.191 & $3.9 \%$ & 0.005 \\
\hline
\end{tabular}

Bold numbers show the highest sensitivity for a case. The rows highlighted in grey show the analysis methods with highest sensitivity

with $\mathrm{SI}_{i, k}$, sensitivity index for index $i$ of analysis method $k ; b_{i, k}$, slope of linear regression linking PAV duration with index $i$ of analysis method $k ; \mathrm{CV}_{i, k}$, mean coefficient of variation for index $i$ of analysis method $k$.

Table 5 sums up the SI for the carbonyl index and the sulfoxide index, respectively. The table contains the slope of the linear regression, the coefficient of determination of the regression $R^{2}$, the mean $\mathrm{CV}$ and the calculated SI. The rows highlighted in grey show the analysis methods with highest sensitivity.
For both indices, the highest sensitivity is derived for the integration and absolute baseline based method on normalized spectrum (IBN, 1.76 for $\mathrm{C}=\mathrm{O}$ and 4.57 for $\mathrm{S}=\mathrm{O}$ ). Using the original spectrum, the most sensitive method is the band maximum and absolute baseline based method for $\mathrm{C}=\mathrm{O}(\mathrm{PBR}, 0.048)$ and the integration and absolute baseline base method for $\mathrm{S}=\mathrm{O}$ (IBR, 0.061).

Since none of the tangential baseline based methods for the sulfoxide index show a linear correlation between index and PAV aging duration, these methods are not considered for sensitivity analysis.
Fig. 4 FTIR ATR spectra from all aging states of $P G$ 76-22 PM

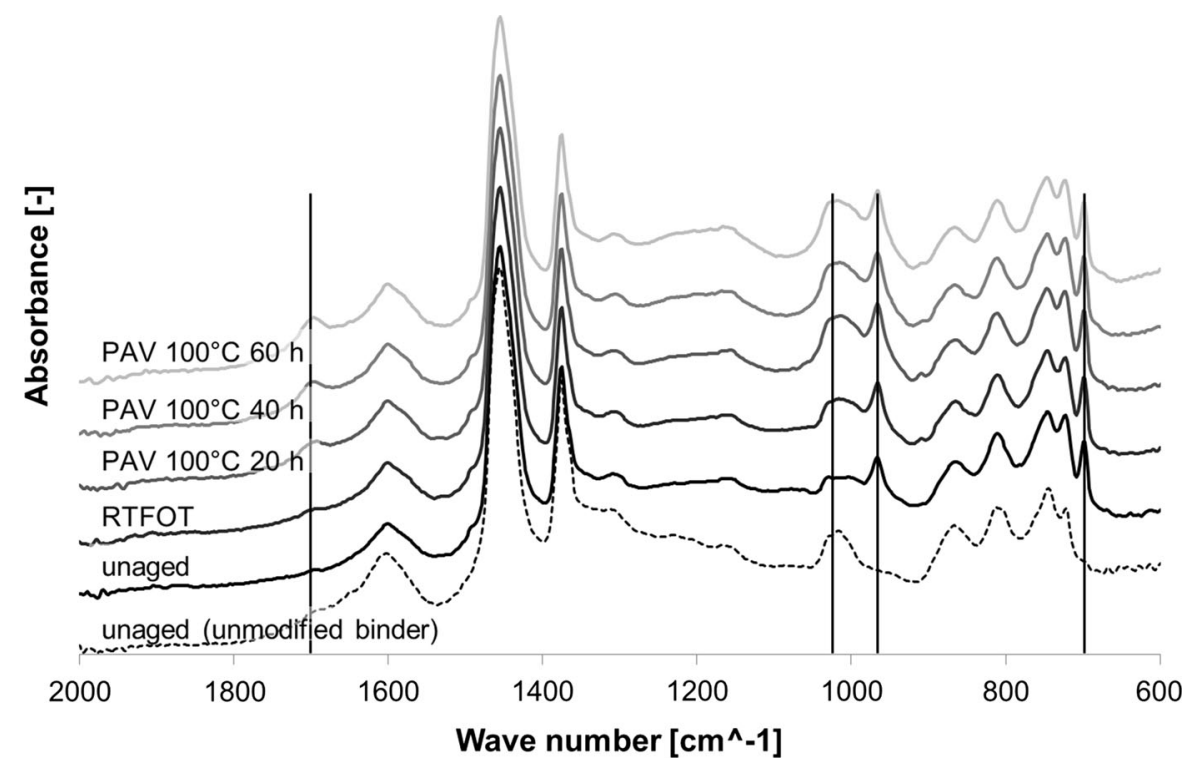



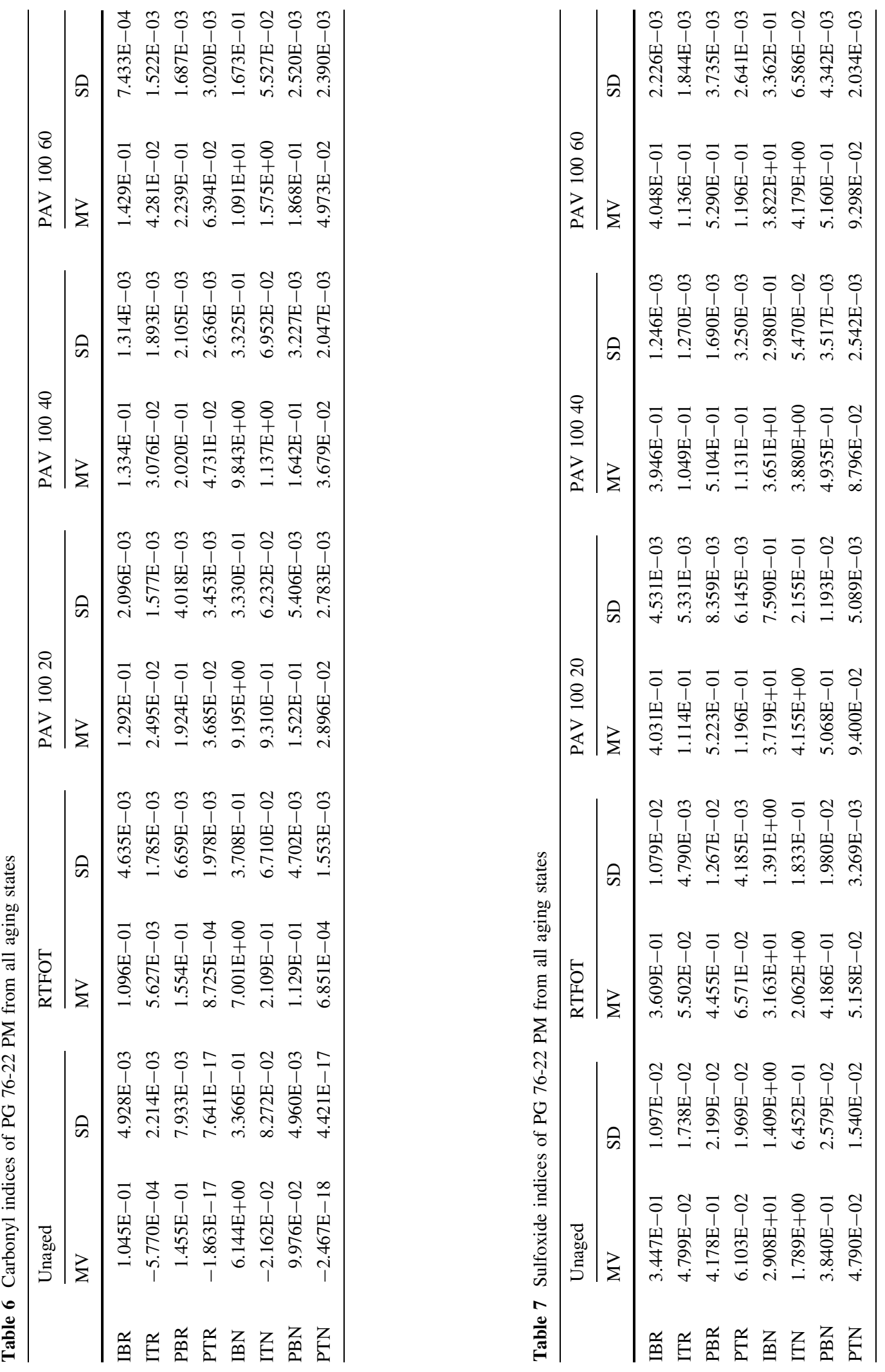
Table 8 Coefficient of variation of carbonyl and sulfoxide indices of PG 76-22 PM for all analysis methods

\begin{tabular}{|c|c|c|c|c|c|c|}
\hline $\mathrm{C}=\mathrm{O}$ & unaged & RTFOT & PAV 10020 & PAV 10040 & PAV 10060 & mean CV \\
\hline IBR & $4.7 \%$ & $4.2 \%$ & $1.6 \%$ & $1.0 \%$ & $0.5 \%$ & $1.8 \%$ \\
\hline ITR & $\mathrm{N} / \mathrm{A}$ & $31.7 \%$ & $6.3 \%$ & $6.2 \%$ & $3.6 \%$ & $11.9 \%$ \\
\hline PBR & $5.5 \%$ & $4.3 \%$ & $2.1 \%$ & $1.0 \%$ & $0.8 \%$ & $2.0 \%$ \\
\hline PTR & $\mathrm{N} / \mathrm{A}$ & $\mathrm{N} / \mathrm{A}$ & $9.4 \%$ & $5.6 \%$ & $4.7 \%$ & $6.6 \%$ \\
\hline IBN & $5.5 \%$ & $5.3 \%$ & $3.6 \%$ & $3.4 \%$ & $1.5 \%$ & $3.5 \%$ \\
\hline ITN & $\mathrm{N} / \mathrm{A}$ & $31.8 \%$ & $6.7 \%$ & $6.1 \%$ & $3.5 \%$ & $12.0 \%$ \\
\hline $\mathrm{PBN}$ & $5.0 \%$ & $4.2 \%$ & $3.6 \%$ & $2.0 \%$ & $1.3 \%$ & $2.8 \%$ \\
\hline PTN & N/A & N/A & $9.6 \%$ & $5.6 \%$ & $4.8 \%$ & $6.7 \%$ \\
\hline $\mathrm{S}=\mathrm{O}$ & unaged & RTFOT & PAV 10020 & PAV 10040 & PAV 10060 & mean CV \\
\hline IBR & $3.2 \%$ & $3.0 \%$ & $1.1 \%$ & $0.3 \%$ & $0.5 \%$ & $1.2 \%$ \\
\hline ITR & $36.2 \%$ & $8.7 \%$ & $4.8 \%$ & $1.2 \%$ & $1.6 \%$ & $4.1 \%$ \\
\hline PBR & $5.3 \%$ & $2.8 \%$ & $1.6 \%$ & $0.3 \%$ & $0.7 \%$ & $1.4 \%$ \\
\hline PTR & $32.3 \%$ & $6.4 \%$ & $5.1 \%$ & $2.9 \%$ & $2.2 \%$ & $4.1 \%$ \\
\hline IBN & $4.8 \%$ & $4.4 \%$ & $2.0 \%$ & $0.8 \%$ & $0.9 \%$ & $2.0 \%$ \\
\hline ITN & $36.1 \%$ & $8.9 \%$ & $5.2 \%$ & $1.4 \%$ & $1.6 \%$ & $4.3 \%$ \\
\hline $\mathrm{PBN}$ & $6.7 \%$ & $4.7 \%$ & $2.4 \%$ & $0.7 \%$ & $0.8 \%$ & $2.2 \%$ \\
\hline PTN & $32.1 \%$ & $6.3 \%$ & $5.4 \%$ & $2.9 \%$ & $2.2 \%$ & $4.2 \%$ \\
\hline
\end{tabular}

Bold numbers show the smallest CV for a case. The rows highlighted grey show the analysis method with smallest mean CV

Table 9 Sensitivity index of carbonyl indices of PG 76-22 PM for all analysis methods

\begin{tabular}{|c|c|c|c|c|c|c|c|c|}
\hline & \multicolumn{4}{|c|}{ Carbonyl Index } & \multicolumn{4}{|c|}{ Sulfoxide Index } \\
\hline & Slope $b_{C O}$ & $\mathrm{R}^{2}$ & mean CV & $\begin{array}{l}\text { Sensitivity } \\
\text { Index }\end{array}$ & Slope $b_{\text {so }}$ & $\mathrm{R}^{2}$ & mean $\mathrm{CV}$ & $\begin{array}{l}\text { Sensitivity } \\
\text { Index }\end{array}$ \\
\hline$\overline{\mathrm{IBR}}$ & 0.00034 & 0.951 & $1.8 \%$ & 0.033 & 0.00004 & 0.025 & $1.2 \%$ & 0.006 \\
\hline ITR & 0.00045 & 0.961 & $11.9 \%$ & 0.008 & 0.00006 & 0.060 & $4.1 \%$ & 0.002 \\
\hline PBR & 0.00079 & 0.952 & $2.0 \%$ & 0.061 & 0.00017 & 0.128 & $1.4 \%$ & 0.019 \\
\hline PTR & 0.00068 & 0.983 & $6.6 \%$ & 0.010 & 0.00000 & 0.000 & $4.1 \%$ & 0.000 \\
\hline IBN & 0.04287 & 0.981 & $3.5 \%$ & 1.507 & 0.02581 & 0.358 & $2.0 \%$ & 2.072 \\
\hline ITN & 0.01610 & 0.959 & $12.0 \%$ & 0.296 & 0.00060 & 0.005 & $4.3 \%$ & 0.022 \\
\hline PBN & 0.00087 & 0.970 & $2.8 \%$ & 0.038 & 0.00023 & 0.163 & $2.2 \%$ & 0.018 \\
\hline PTN & 0.00052 & 0.980 & $6.7 \%$ & 0.008 & -0.00003 & 0.025 & $4.2 \%$ & -0.001 \\
\hline
\end{tabular}

Bold numbers show the highest sensitivity for a case. The rows highlighted in grey show the analysis methods with highest sensitivity

\subsection{Validation}

Figure 4 shows examples of FTIR spectra from PG 76-22 PM in all states of aging. In addition, the dotted line on the bottom of the diagram shows the FTIR spectrum of the unmodified PG 64-16 sample. Again, the increase in oxidation with aging in the carbonyl and sulfoxide areas is visible for the SBS modified sample. In addition, two further peaks are marked in the spectra, which are characteristic of SBS modified samples: at $699 \mathrm{~cm}^{-1}$ the styrene band and at $966 \mathrm{~cm}^{-1}$ the butadiene band is visible. Especially the butadiene band needs to be taken into consideration since it interferes with the sulfoxide band. Thus, the wavenumber limits for the sulfoxide area have to 
be adjusted to prevent any impact of the butadiene band on the sulfoxide index:

- Carbonyl: 1666-1746 $\mathrm{cm}^{-1}$

- Sulfoxide: $984-1066 \mathrm{~cm}^{-1}$

- Reference (aliphatic): $1319-1520 \mathrm{~cm}^{-1}$

The full set of results (mean values and standard deviation) for all aging states and applied analysis methods can be found in Table 6 for the carbonyl index and in Table 7 for the sulfoxide index, respectively.

Table 8 presents the CVs of all analysis methods for the carbonyl and sulfoxide indices, respectively. In line with the results of the PG 64-16 (Table 4), the most repeatable method using original spectra is the IBR method, followed by the PBR method. When using normalized spectra, the most repeatable method is the PBN method, followed by the IBN method. And similar to the PG 64-16 binder, the worst repeatability can be found for the ITR and ITN methods.

Table 9 contains the results of the sensitivity index according to Eq. (7) for the PG 76-22 PM. For the carbonyl index, the results validate what has been shown for the PG 64-16 sample above. Using original spectra, the PBR method shows the highest sensitivity to changes in the carbonyl index due to aging. For normalized spectra, the IBN method is the most sensitive one. The coefficient of determination $R^{2}$ is above 0.95 for all applied methods. Thus, a linear correlation between increasing aging time in PAV and increase in oxidative structures is obvious.

The situation is more complex for the sulfoxide index. For none of the methods, a linear correlation between sulfoxide index and PAV aging duration can be established. The coefficient of determination is below 0.35 for all cases. This is in contrast to the PG 64-16 results where strong linear correlations were found for the methods using the absolute baseline. The most likely explanation for the difference between PG 64-16 and the SBS modified PG 76-22 PM is proximity of the sulfoxide $\left(1030 \mathrm{~cm}^{-1}\right)$ and butadiene $\left(966 \mathrm{~cm}^{-1}\right)$ bands. As shown in Fig. 4, both peaks interfere and thus, a separate analysis of both structures does not seem to be possible in case of SBS modified binders. For this reason, the sulfoxide index of PG 76-22 PM is not taken into consideration for the sensitivity analysis.

\section{Summary and recommendations}

This study works towards recommendations on how to analyze FTIR ATR spectra from bituminous binders with high repeatability and sensitivity in terms of changes due to oxidative aging. Two binder samples, a PG 64-16 and an SBS modified PG 76-22 PM, were aged by RTFOT and PAV for 20,40 and $60 \mathrm{~h}$ and FTIR ATR spectra were taken from the unaged and all aged binder samples. For each aging state, 10 individual PG 64-16 samples were analyzed in FTIR and 9 spectra were taken for each sample. Thus, 90 spectra were available for analysis for each aging state. For validation purposes, 5 individual PG 76-22 PM samples were analyzed in FTIR with 9 spectra per sample. Eight different analysis methods were taken into consideration. The methods work either with the original or a normalized spectrum, absolute or tangential baseline and use a band maximum or integration based index. The spectra were analyzed in terms of changes in the carbonyl and sulfoxide bands, which are considered as the main structural changes due to oxidative aging. To assess the repeatability of each analysis method, the coefficient of variation (CV) was taken as a benchmark parameter. To study the sensitivity of the different analysis methods to changes in the carbonyl and sulfoxide index, a sensitivity index was introduced. This index is based on the slope of the linear regression of the carbonyl/sulfoxide index versus aging duration in PAV and the $\mathrm{CV}$ of each analysis method. Based on the findings, the following recommendations can be given:

- In terms of repeatability, the methods based on original spectra using the absolute baseline show the smallest $\mathrm{CV}$ and thus the best repeatability. This is true for both, the carbonyl and sulfoxide index. For methods based on normalized spectra, again, the ones using the absolute baseline produce best repeatability. Both, approaches working with band maxima and integration are equally repeatable.

- In terms of sensitivity, the integration based method with normalized spectrum and absolute baseline show the highest sensitivity to changes in the carbonyl/sulfoxide index. Based on sensitivity considerations, no method based on tangential base line can be recommended. 
- These conclusions based on the unmodified binder results have been validated and are supported by the SBS modified binder results, with one exemption: Due to the proximity and thus, interference between the sulfoxide and butadiene band, the analysis of changes in the sulfoxide structures seems to be biased by the SBS modification.

Thus, for future analysis of FTIR ATR spectra of bituminous binders to investigate changes in the oxidative aging, it is recommended to work with normalized spectra, use an absolute baseline and work with integration of areas for index production in favor over peak based methods. This study is limited to laboratory aged binder samples using aging methods that trigger oxidative aging. Field aging under real climate conditions is by far more complex since other effects, most prominently UV radiation and moisture, affect aging behavior of bituminous binders. However, laboratory aged binder samples were employed in this study due to the controlled environment throughout the aging procedures.

To further validate the findings of this study, the next part of the research should include a round robin study including multiple different laboratories using different devices. This way, urgently needed information on reproducibility of FTIR ATR working with bituminous binders could be gathered as well. Also, a study including field aged samples is planned as well, to transfer findings from lab to field.

Acknowledgement Open access funding provided by TU Wien (TUW). The authors would like to express their gratitude to the California Department of Transportation (Caltrans) and the Max Kade Foundation for financial support of this study. The contents of this paper reflect the views of the authors and do not necessarily reflect the official views or policies of the supportive organizations.

Funding The study was partly funded by the California Department of Transportation (Caltrans) and the Max Kade Foundation.

\section{Compliance with ethical standards}

Conflict of interest The authors declare that they have no conflict of interest.

Open Access This article is distributed under the terms of the Creative Commons Attribution 4.0 International License (http:// creativecommons.org/licenses/by/4.0/), which permits unrestricted use, distribution, and reproduction in any medium, provided you give appropriate credit to the original author(s) and the source, provide a link to the Creative Commons license, and indicate if changes were made.

\section{References}

1. Palade LI, Attane P, Camaro S (2000) Linear viscoelastic behavior of asphalt and asphalt based mastic. Rheol Acta 39(2):180-190

2. Garcia-Morales M et al (2004) Linear viscoelasticity of recycled EVA-modified bitumens. Energy Fuels 18(2):357-364

3. Costanzi M, Cebon D (2015) Generalized phenomenological model for the viscoelasticity of bitumen. J Eng Mech 141(5):04014160

4. Hofko B et al (2015) Alternative approach toward aging of bitumen and asphalt mixes. In: TRB 94th Annual Meeting Compendium of Papers, $\mathrm{p} 15$

5. Eberhardsteiner L et al (2014) Towards a microstructural model of bitumen aging behavior. Int $\mathrm{J}$ Pavement Eng 16(10):939-949

6. Romberg JW, Traxler RN (1947) Rheology of asphalt. J Colloid Sci 2(1):33-47

7. Brodnyan JG et al (1960) The rheology of asphalt. 3. Dynamic mechanical properties of asphalt. Trans Soc Rheol 4:279-296

8. Kim HS, Lee SJ, Amirkhanian S (2010) Rheology investigation of crumb rubber modified asphalt binders. KSCE J Civil Eng 14(6):839-843

9. Merusi F, Giuliani F (2011) Rheological characterization of wax-modified asphalt binders at high service temperatures. Mater Struct 44(10):1809-1820

10. Naskar M et al (2013) Effect of ageing on different modified bituminous binders: comparison between RTFOT and radiation ageing. Mater Struct 46(7):1227-1241

11. Asgharzadeh SM et al (2015) Evaluation of rheological master curve models for bituminous binders. Mater Struct 48(1-2):393-406

12. Roman C, Garcia-Morales M (2017) Linear rheology of bituminous mastics modified with various polyolefins: a comparative study with their source binders. Mater Struct 50(1):86

13. Chen JS, Huang LS (2000) Developing an aging model to evaluate engineering properties of asphalt paving binders. Mater Struct 33(233):559-565

14. Di Benedetto $\mathrm{H}$ et al (2004) Fatigue of bituminous mixtures. Mater Struct 37(267):202-216

15. Dai QL, You ZP (2008) Micromechanical finite element framework for predicting viscoelastic properties of asphalt mixtures. Mater Struct 41(6):1025-1037

16. Delgadillo R, Bahia HU, Lakes R (2012) A nonlinear constitutive relationship for asphalt binders. Mater Struct 45(3):457-473

17. Eberhardsteiner L et al (2014) Influence of asphaltene content on bitumen behavior: experimental investigation and micromechanical modeling. Mater Struct 48(10):3099-3112

18. Das PK et al (2015) On the oxidative ageing mechanism and its effect on asphalt mixtures morphology. Mater Struct 48(10):3113-3127 
19. Perraton D et al (2016) 3Dim experimental investigation of linear viscoelastic properties of bituminous mixtures. Mater Struct 49(11):4813-4829

20. Glotova NA et al (1980) Changes in rheological properties and chemical-composition of asphalts upon aging. Chem Technol Fuels Oils 16(3-4):278-280

21. Ali MF, Siddiqui MN (2001) Changes in asphalt chemistry and durability during oxidation and polymer modification. Pet Sci Technol 19(9-10):1229-1249

22. Gawel I, Baginska K (2004) Effect of chemical nature on the susceptibility of asphalt to aging. Pet Sci Technol 22(9-10):1261-1271

23. Xu GJ, Wang H (2016) Study of cohesion and adhesion properties of asphalt concrete with molecular dynamics simulation. Comput Mater Sci 112:161-169

24. Kumbargeri YS, Biligiri KP (2016) Rational performance indicators to evaluate asphalt materials' aging characteristics. J Mater Civil Eng 28(12):04016157

25. Taylor R et al (1998) Rheological and structural analysis of recovered asphalt binders from the FHWA ALF rutting and fatigue experiments, Part III FTIR spectral analysis. In Abstracts of papers of the American chemical society, vol 216, pp U847

26. Mothe MG, Leite LFM, Mothe CG (2008) Thermal characterization of asphalt mixtures by TG/DTG, DTA and FTIR. J Therm Anal Calorim 93(1):105-109

27. Zhang F, Yu JY, Han J (2011) Effects of thermal oxidative ageing on dynamic viscosity, TG/DTG, DTA and FTIR of SBS- and SBS/sulfur-modified asphalts. Constr Build Mater 25(1):129-137

28. Cao XJ, Lei YB, Wang W (2011) Study on thermal oxidation of asphalt fractions by in situ FTIR analysis. Mater Sci Eng Appl 160-162:330-335

29. Bowers BF et al (2014) Investigation of reclaimed asphalt pavement blending efficiency through GPC and FTIR. Constr Build Mater 50:517-523
30. Marsac P et al (2014) Potential and limits of FTIR methods for reclaimed asphalt characterisation. Mater Struct 47(8):1273-1286

31. Yang X, You ZP, Mills-Beale J (2015) Asphalt binders blended with a high percentage of biobinders: aging mechanism using FTIR and rheology. J Mater Civil Eng 27(4):04014157

32. Nivitha MR, Prasad E, Krishnan JM (2016) Ageing in modified bitumen using FTIR spectroscopy. Int J Pavement Eng 17(7):565-577

33. Feng ZG et al (2016) FTIR and rheology analysis of aging on different ultraviolet absorber modified bitumens. Constr Build Mater 115:48-53

34. Feng ZG et al (2016) FTIR analysis of UV aging on bitumen and its fractions. Mater Struct 49(4):1381-1389

35. Smith BC (2011) Fundamentals of fourier transform infrared spectroscopy. CRC Press, Boca Raton

36. Liu M et al (1998) Oxygen uptake as correlated to carbonyl growth in aged asphalts and asphalt Corbett fractions. Ind Eng Chem Res 37(12):4669-4674

37. CEN, EN 12607-1 (2007) Bitumen and bituminous binders: determination of the resistance to hardening under the influence of heat and air-Part 1: RTFOT method. Brussels

38. CEN, EN 14769 (2012) Bitumen and bituminous binders: accelerated long-term ageing conditioning by a pressure ageing vessel (PAV). Brussels

39. QuestChemicalCorporation (2017) Orange Solv Plus: technical data report-Product \# 378

40. Lamontagne J et al (2001) Comparison by Fourier transform infrared (FTIR) spectroscopy of different ageing techniques: application to road bitumens. Fuel 80(4):483-488 